\title{
A la recherche de la protéine de la mucoviscidose
}

Les nouvelles

de ce numéro ont été préparées par : J.-C. Dreyfus

A. Kahn

J.-P. Grünfeld

\section{DERNIÈRE HEURE}

Dans les numéros de Nature du 28 Novembre et de Lancet du 30 Novembre sont parus quatre articles localisant le gène de la mucoviscidose sur le bras long du chromosome 7 , probablement en q22.

1. Voir lc numciro enticr de Prenatal Diagnosis, Mars 1985 .

2. Pediat Res 1975; $9: 635-640$. Références citée dans Nature [4].

3. L.ancet 1980; i : 330-331. Référence citćc dans Nature [4].

4. Van Heyningen, V., Hayward, C., Fletcher, J. McAulcy, C. 'Tissuc localization and chromosomal assignment of a scrum protein that tracks the

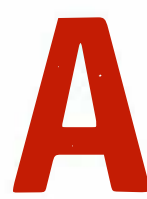

vec un cas sur 2000 naissances, soit une fréquence du gène supérieure à 1 sur 25 , la mucoviscidose, ou fibrose kystique, est la maladie génétique à transmission autnsomique récessive la plus fréquente dans nos pays.Les signes $b$, logiques (test de la sueur), comme les méthodes actuellement :ppliquées à son diagnostic prénatal [1] n'en représentent que des manifestations indirectes. Mais voici qu'un symptôme biochimique prétend la caractériser. Il s'agit d'une protéine décrite en 1975 par Wilson et al. [2] pour laquelle Manson et Brock [3] ont mis en œuvre un test immunologique qui en démontre la présence dans le sérum des sujets homozygotes et, à un taux moitié moindre, dans celui des hétérozygotes. Cet antisérum de cobaye est difficile à obtenir, la reproductibilité des résultats est médiocre. Les tentatives de purification à partir du sérum des malades ont échoué du fait de la labilité de la protéine et d'une forte contamination par de la sérumalbumine. On a donc cherché une autre source. V. van Heyningen $e t$ al. à Edimbourg [4] ont observé que des granulocytes normaux (mais surtout ceux de la leucémie myéloìde chronique-LMC) possèdent une protèine qui ne se distingue pas immunologiquement de ce qu'ils nomment " protéine de la fibrose kystique". Or, il existe une lignée de cellules myéloïdes de souris (WEHI-TG) qui peut former des hybrides somatiques avec des cellules de LMC humaine, capables d'exprimer certaines protéines de ces dernières. En sous-clonant ces hybrides et en utilisant un antisérum de cobaye, les auteurs ont pu démontrer que la protéine étudiée est codée par un gène porté par le chromosome I humain.

Nous nous trouvons donc actuellement dans une situation paradoxale : une protéine reconnue par un antisérum spécifique et dont le gène a pu être localisé avec précision est présente dans les granulocytes de LMC mais aussi, à un taux moindre, dans les polynucléaires normaux et, peut-être, dans d'autres tissus; dans le sérum en revanche, la présence de cette protéine semble signer celle du gène de la mucoviscidose et permet, en la quantifiant, de distinguer entre homo- et hétorozygotes.

La plupart des interprétations ne résistent pas à la critique. Celle des auteurs est la suivante : l'antigène serait une protéine normalement synthétisée par l'organisme mais elle serait un précurseur destiné à disparaître sous l'effet d'une réaction protéolytique le transformant en un produit probablement biologiquement actif. Dans la fibrose kystique, cette protéine serait altérée dans sa structure de façon telle que le processus protéolytique ne puisse avoir lieu; c'est l'absence du produit final qui serait à la base des anomalies cliniques et biologiques. Chez l'hétérozygote, la même altération empêcherait la disparition de la protéine du sérum mais la présence d'une dose de protéine normale suffirait à prévenir toute conséquence clinique. Il n'y a là encore qu'une hypothèse que la recherche d'anticorps monoclonaux ainsi que des essais de production d'une sonde d'ADN complémentaire s'efforcent d'étayer. Compte tenu de l'importance de cette maladie, nous avons souhaité rapporter ces résultats car la piste proposée suit un des très rares fils conducteurs actuellement plausibles. 